\title{
The Distribution and Source of MRDOs Infection: A Retrospective Study in 8 ICUs, 2013-2019
}

\author{
Zhan-jie $\mathrm{Li}^{1}{ }^{1} *$ \\ Ke-wei Wang ${ }^{2, *}$ \\ Bo Liu' \\ Feng Zang' \\ Yu Zhang ${ }^{3}$ \\ Wei-hong Zhang' \\ Su-ming Zhou ${ }^{4}$ \\ Yong-xiang Zhang' \\ 'Department of Infection Control, The \\ First Affiliated Hospital of Nanjing \\ Medical University, Nanjing, 210029, \\ Jiangsu, People's Republic of China; \\ ${ }^{2}$ Institute of Integrated Traditional \\ Chinese and Western Medicine, Affiliated \\ Hospital of Jiangnan University, Wuxi, \\ 214062, Jiangsu, People's Republic of \\ China; ${ }^{3}$ Department of Infection \\ Management, the Second Affiliated \\ Hospital of Xuzhou Medical University, \\ Xuzhou, 221006, Jiangsu, People's \\ Republic of China; ${ }^{4}$ Department of \\ Geriatric Critical Medicine, The First \\ Affiliated Hospital of Nanjing Medical \\ University, Nanjing, 210029, Jiangsu, \\ People's Republic of China \\ *These authors contributed equally to \\ this work
}

Correspondence: Yong-xiang Zhang Department of Infection Control, First Affiliated Hospital of Nanjing Medical University, No. 300 Guangzhou Road, Nanjing, 210029 , Jiangsu, People's

Republic of China

Tel +86-025-683069l7;

$+86-13951951616$

Email Yongxiang21602IICU@163.com

Su-ming Zhou

Department of Geriatric Critical

Medicine, The First Affiliated Hospital of

Nanjing Medical University, No. 300

Guangzhou Road, Nanjing, 210029 ,

Jiangsu, People's Republic of China

Email zhousmco@aliyun.com
Background: To analyze the distribution and source of MDROs infection in the ICUs and to provide a basis for formulating more effective prevention and control programs for MDROs.

Methods: A retrospective investigation was conducted on MDROs infection in 8 ICUs of a large tertiary hospital from July 2013 to June 2019. A total of 2629 strains of MDROs isolated from 1701 inpatients were selected for analysis. The MDROs of the 8 ICUs were divided into two types of four categories according to source: out-of-hospital (out-of-hospital transfer and community acquisition) and in-hospital (in-hospital transfer and department acquisition) infections.

Results: CRAB (41.84\%) and CRE (35.07\%) accounted for the majority of the infecting MDROs. The detection rates of MRSA, CRAB, CRPA and CRE were $61.24 \%, 83.75 \%$, $43.01 \%$ and $30.15 \%$, respectively. The top three infection sites of MDROs were the lower respiratory tract $(81.10 \%)$, blood $(6.70 \%)$ and abdominal cavity $(5.80 \%)$. The out-of-hospital and in-hospital infection rates of MDROs were 50.51\% and 49.49\%, respectively; the out-ofhospital infection rates for MRSA, CRAB, CRPA and CRE were 43.56\%, 55.91, 64.44\% and $44.58 \%$, respectively. The proportions of MRSA, CRAB, CRPA and CRE infections contracted in the department were $40.98 \%, 36.27 \%, 25.56 \%$ and $46.62 \%$, respectively. There was a statistically significant difference between comprehensive ICU and specialized ICU wards as sources for CRAB infections $(\mathrm{P}<0.001)$.

Conclusion: The main source of MDROs in the ICU is not the hospital itself entirely. It is particularly important to strengthen the identification of MDRO sources and implement more effective and accurate infection prevention and control measures.

Keywords: MDRO, intensive care unit, ICU, source

\section{Key Points}

More than half of the infected MDROs in ICU comes from out-of-hospital transfer and community acquisition.Different ICU have different sources of MDROs infection.

\section{Introduction}

The intensive care unit (ICU) is a special department where critically ill patients are treated; there is a high risk of hospital infection, and these sites are the focus of hospital infection supervision. ${ }^{1}$ Multidrug-resistant organisms (MDROs), as the main pathogens of hospital-acquired infection, ${ }^{2}$ easily cause outbreaks of hospital infection when not properly managed. ${ }^{3}$ MDROs have also become a major difficulty in anti-infection treatment. Indeed, there has been a continuous increase in 
MDRO infection in different countries and regions in recent years. ${ }^{4}$ The widespread use of broad-spectrum antibiotics has resulted in an increase in MDROs in the ICU worldwide, ${ }^{5-7}$ which has been a growing challenge for clinicians. $^{8-10}$ It has been reported that more than onethird of patients who are critically ill in medical or surgical ICUs are infected ${ }^{11}$ and that $35 \%$ of infected patients in the ICU are at particular risk for MDRO infection. ${ }^{12}$ Moreover, studies have shown that the detection rate of MDROs in the ICU is higher than that in general wards. ${ }^{11}$

As the "severe disaster area" of MDROs, the ICU has become the top priority of MDRO prevention and control in hospitals. However, whether the serious MDRO problem is due to inadequate implementation of prevention and control measures in the ICU or to other reasons is worthy of discuss. Despite many studies both at home and abroad on MDRO detection and infection in the ICU, few studies have examined the source of MDROs in the ICU. This study collected data from hospitalized patients with MDROs infection in eight ICUs of a hospital from July 2013 to June 2019 and conducted a retrospective investigation and analysis of the source of MDRO infection.

\section{Methods}

\section{Study Setting and Patient Population}

The study included 1701 inpatients with 2629 MDROs in eight different ICUs of the First Affiliated Hospital of Nanjing Medical University (Nanjing, China) from July 2013 to June 2019. The 8 ICUs included the thoracic surgery ICU, cardiothoracic ICU, neurosurgery ICU, surgical trauma ICU, geriatric ICU, Integrated ICU (ICU), emergency ICU and respiratory ICU.

Inclusion criteria: (1) patients in ICU departments; (2) Detect MDRO; (3) MDRO infection. Exclusion criteria: (1) patients in non-ICU departments; (2) MDRO was not detected; (3) MDRO colonization or contamination. Elimination of repetitive strains in the same part of the same patient. This study was approved by the hospital ethics committee and exempted from informed consent (2019-SR-075).

\section{Bacterial Identification and Drug Susceptibility Testing}

Bacterial identification was conducted using a Vitek-2 compact automatic bacterial identification instrument or an API identification system. A bacterial drug sensitivity test was conducted using the Vitek-2 compact automatic bacterial identification instrument or the disk diffusion method. ${ }^{13}$ Drug susceptibility was assessed according to the American Society for Clinical and Laboratory Standardization (CLSL) Standard (2017). ${ }^{14}$ The quality control strains used were Escherichia coli (ATCC 25922), Klebsiella pneumoniae (ATCC 700603), Staphylococcus aureus (ATCC 25923), Acinetobacter baumannii (ATCC 19606), Pseudomonas aeruginosa (ATCC 278553) and Enterobacter cloacae (ATCC 700323), which were obtained from the Clinical Testing Center of National Health Commission of the People's Republic of China.

\section{Research Methods}

A retrospective investigation was conducted, and MDRO infections (including MRSA (Methicillin-resistant Staphylococcus Aureus), CRE (Carbapenem - resistant Enterobacteriaceae), CRAB (Carbapenem-resistant Acinetobacter Baumannii), and CRPA (Carbapenemresistant Pseudomonas Aeruginosa)) in 8 ICUs of the hospital from July 2013 to June 2019 were assessed using the Xinlin hospital infection real-time monitoring system. The full-time Infection management professionals of the hospital examine each item in the medical records of patients with this type of infection and determine the sources of the MDROs, as based on the standard for identifying nosocomial infections. The MDROs infection source was divided into two types of four categories: in-hospital infection (in-hospital transfer, department acquisition) and out-of-hospital infection (out-ofhospital transfer, community acquisition). To study the influence of different types of ICUs on the source of MDROs, the 8 ICUs were divided into comprehensive ICUs (including ICU, emergency ICU and geriatric ICU) and specialized ICUs (including respiratory ICU, surgical trauma ICU, neurosurgery ICU, cardiothoracic ICU and thoracic surgery ICU), and the difference source of MDROs between the comprehensive and specialized ICUs was analyzed.

\section{Diagnostic Criteria}

Cases of infection were determined according to the 'Diagnostic Standards for Hospital Infections' decreed by the Chinese Ministry of Health (2001). ${ }^{15}$ Patients with MDRO were evaluated using the international expert proposal for interim standard definitions for acquired resistance. $^{16}$ 


\section{Outcome Variables}

In this study, MDRO cases included both in-hospital and out-of-hospital infections. In-hospital infections included in-hospital transfers, whereby Infection occurred in other department of our hospital for more than 48 hours before transfer to ICU, or within 48 hours after transfer to ICU in other departments of our hospital, and department acquisition, whereby infection occurred for more than $48 \mathrm{~h}$ after being transferred to the ICU or less than $48 \mathrm{~h}$ after being out of the ICU in our hospital. Out-of-hospital infections included out-hospital transfer, whereby infection occurred for more than $48 \mathrm{~h}$ in another hospital, and community acquisition, whereby infection occurred for less than $48 \mathrm{~h}$ in another hospital or not hospitalized in other hospitals.

The following formula was applied: Detection rate of MDRO $=$ the number of multiple MDRO detected (including infection and colonization)/the total number of strains detected by the pathogen in the same period $\times 100 \%$.

\section{Statistical Analysis}

Continuous variables are presented as means \pm standard deviation (SD). Proportions were calculated as percentages of patients with available data. Chi-square tests or Fisher's exact probability tests were used to compare categorical data. A two-tailed $\mathrm{P}$ value $<0.05$ was considered statistically significant. Statistical analyses were performed with
SPSS 22.0 software (IBM SPSS, Chicago, USA) and GraphPad Prism 7 (GraphPad Software, La Jolla, CA).

\section{Results \\ Distribution of MDROs}

A total of 1701 inpatients infected with MDROs were included in the study, with a mean of 61.1 years (Range: 8-98 years, $\bar{X}: 61.39 \pm 17.02$ ). There were 1213 male patients (71.31\%) and 488 female patients (28.69\%). A total of 2629 MDRO strains were collected in the eight ICUs; ICU (830/ 2629 [31.57\%]) had the most MDROs, followed by geriatric ICUs (521/2629 [19.82\%]), neurosurgery ICU (489/2629 [18.60\%]), emergency ICU (300/2629 [11.41\%]), cardiothoracic ICU (180/2629 [6.85\%]), respiratory ICU (164/ 2629 [6.24\%]), and surgical trauma ICU (131/2629 [4.98\%]). MDROs infection was mainly caused by CRAB (1100/2629 [41.84\%]), followed by CRE (922/2629 [35.07\%]), MRSA (427/2629 [16.24\%]), and CRPA (180/ 2629 [6.85\%]). Similarly, CRAB and CRE were the main MDROs in ICU, respiratory ICU, emergency ICU, geriatric ICU, surgical trauma ICU, neurosurgery ICU, and cardiothoracic ICU ICU. However, MRSA was the main MDRO in the thoracic surgery ICU (Figure 1).

\section{Detection Rate of MDROs}

The detection rate for the four MDROs in 8 ICUs are presented in Table 1. For the four MDROs combined,

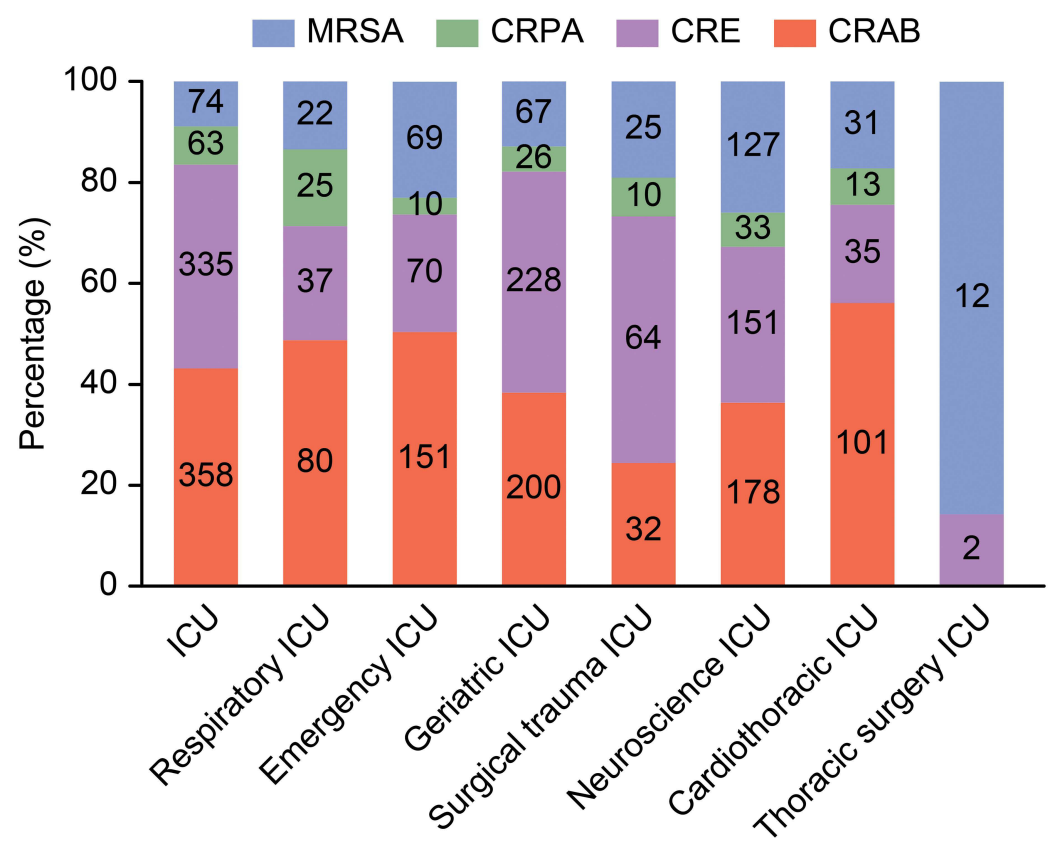

Figure I Distribution of MDRO-infected strains in $8 \mathrm{ICU}$ wards. 


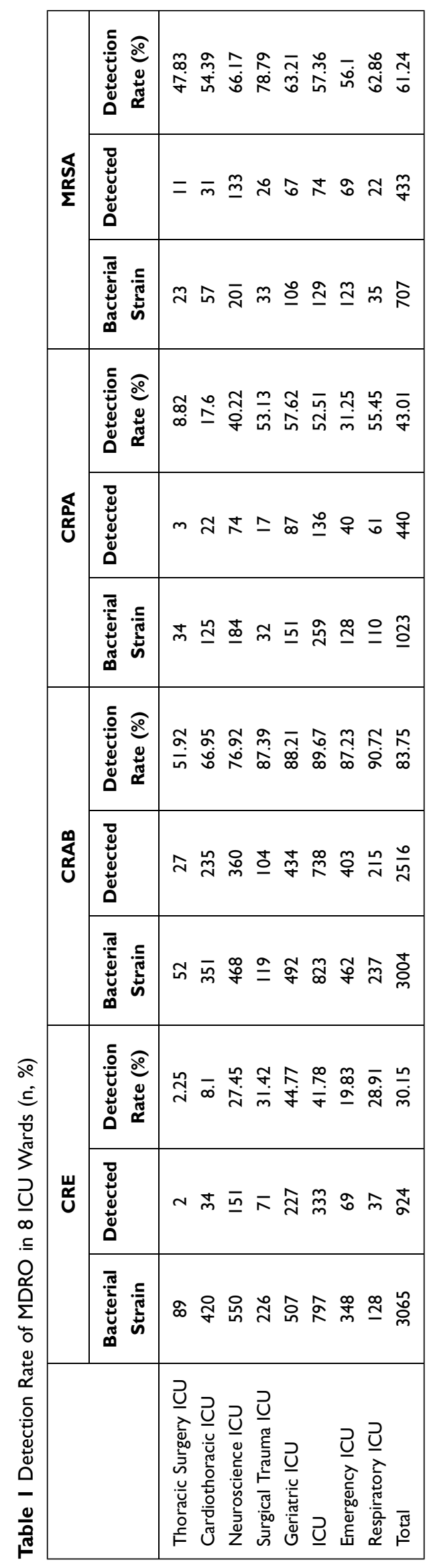

CRAB showed the highest detection rate ( $83.75 \%)$, followed by MRSA (61.24) and CRPA (43.01\%), with CRE having the lowest detection rate $(30.15 \%)$. In addition, detection rates were significantly different $(\mathrm{P}<0.05)$ for MDROs between the four groups. CRAB and CRE is the most commonly detected MDRO, with rates in six ICUs above $75 \%$ and in two ICUs above $40 \%$. The four most common ICUs in which CRPA was detected were the geriatric ICU (57.62\%), respiratory ICU (55.45\%), surgical trauma ICU (53.13\%) and ICU (52.51\%). The highest detection rates of MRSA were found in the surgical trauma ICU $(78.79 \%)$, followed by the neurosurgery ICU (66.17\%), geriatric ICU (63.21\%), respiratory ICU $(62.86 \%)$ and ICU $(57.36 \%)$, with the thoracic surgery ICU (47.83\%) exhibiting the lowest rate.

\section{MDRO Infection Sites}

The main tissue specimens for detecting MDRO were sputum, blood, urine, bile, pus and secretions from the lower respiratory tract, bloodstream, abdominal cavity, urinary tract, skin tissue, cranial cavity, operative sites, and pleural cavity, among others. The most common infection site was the lower respiratory tract $(81.10 \%)$, followed by the blood (6.70\%) and abdominal cavity $(5.80 \%)$ (Figure 2).

\section{Source of MDROs}

Of the 2629 strains of MDROs isolated from inpatients in the eight ICUs, 1328 (50.51\%) were out-of-hospital infections (including out-of-hospital transfers $(756,28.76 \%)$ and community acquisition (572, 21.76\%); 1301 $(49.49 \%)$ were in-hospital infections (including inhospital transfers $(253,9.62 \%)$ and department acquisition (1048, 39.86\%).

Strains of MRSA of out-of-hospital infections accounted for $43.56 \%$ (including out-of-hospital transfers $(25.06 \%)$ and community acquisition (18.50\%)) of all MRSA cases, with $56.44 \%$ being attributed to in-hospital infection (including in-hospital transfers (15.46\%) and department acquisition (40.98\%)). MRSA from out-ofhospital transfers was most commonly $(59.09 \%)$ observed in the respiratory ICU, and MRSA from in-hospital transfers was the leading source $(66.67 \%)$ of MDROs in the thoracic surgery ICU. MRSA from department acquisition substantially accounted for MRSA isolated from inpatients in several ICUs, with the exception of the respiratory ICU (Figure 3A). CRAB strains from out-of-hospital infections accounted for $55.91 \%$ (including out-of-hospital transfers (28.45\%) and community acquisition (27.45\%)) among all 

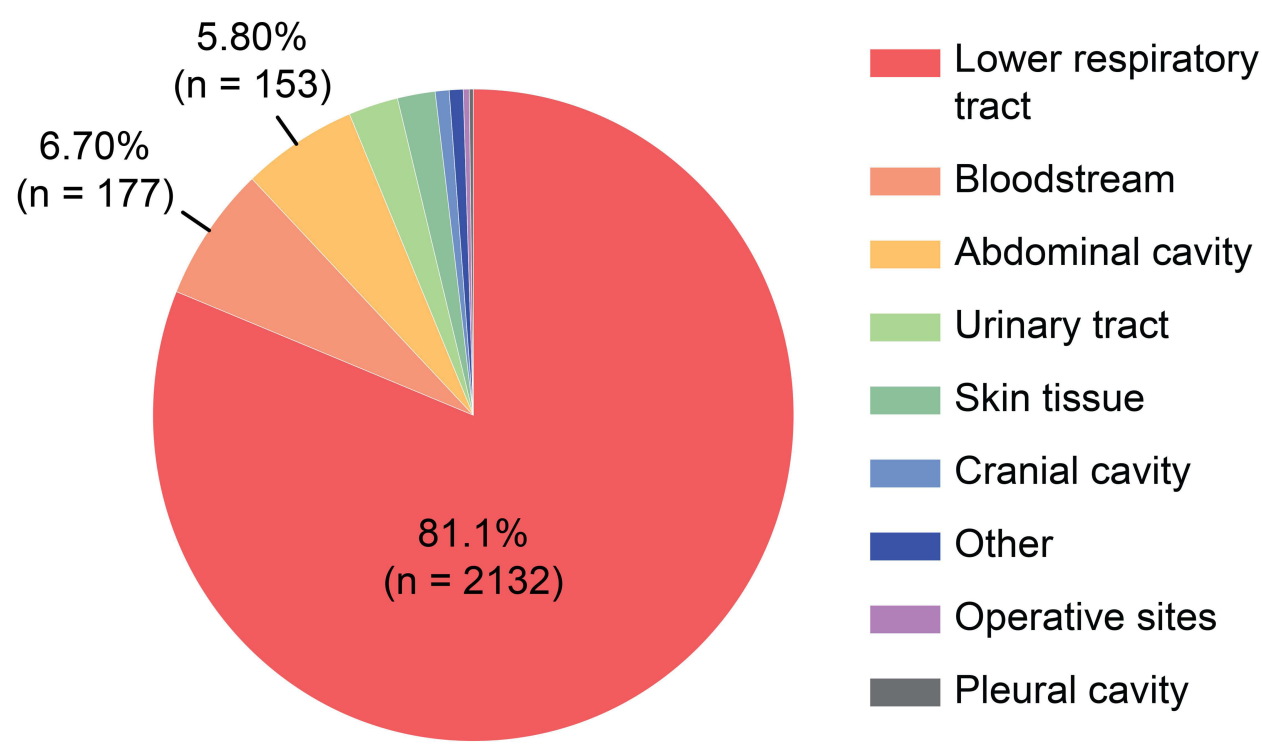

Figure 2 The distribution of MDRO infection sites in 8 ICU wards (\%).

CRAB cases, with $44.09 \%$ being attributed to in-hospital infection (including in-hospital transfers (7.82\%) and department acquisition (36.27\%)) (Figure 3B). As with MRSA, CRE strains from out-of-hospital infections accounted for $44.58 \%$ (including out-of-hospital transfers (28.64\%) and community acquisition (15.94\%)) among all CREs, which was lower than the rate for in-hospital infection, which accounted for $55.42 \%$ (including in-hospital transfers (9.00\%) and department acquisition (46.42\%)) (Figure 3C). Similar to CRAB, strains of CRPA from outof-hospital infections accounted for $64.44 \%$ (including out-of-hospital transfers $(40.00 \%)$ and community acquisition (24.44\%)) among all CRPAs, higher than for inhospital infection, which accounted for $35.56 \%$ (including in-hospital transfers $(10.00 \%)$ and department acquisition $(25.56 \%))$ (Figure 3D).

\section{MDRO Infection Sources in the Comprehensive ICU and Specialist ICU}

There were significant differences in the composition of infection sources of MRSA, CRAB, CRPA and CRE between the comprehensive ICU and specialist ICU (P $<0.001)$. However, there was no significant difference between comprehensive and specialist ICUs with regard to MRSA, CRPA and CRE infection sources. Among the sources of CRAB infection, the ratio of department acquisition in the specialist ICU was significantly higher than that in the comprehensive ICU $(\mathrm{P}<0.05)$ (Table 2).

\section{Discussion}

Acquired infection in the ICU is an important health problem worldwide. ${ }^{17}$ Some risk factors for nosocomial infection and susceptible populations converge in the ICU, where patients are critically ill and at high risk of MDRO infection. ${ }^{18}$ Therefore, the prevention and control of MDROs in the ICU is an important issue that must be solved. To reduce MDRO infection, it is necessary to determine the distribution and sources. In the past 10 years, carbapenems have been considered the last line of defense for the treatment of drug-resistant gram-negative bacterial infections. Carbapenem-resistant strains, especially among Klebsiella pneumoniae, Pseudomonas aeruginosa and Acinetobacter baumannii, have increased rapidly, posing difficulties with regard to clinical antiinfection treatments. ${ }^{19}$

This study shows that CRAB (41.84\%) and CRE (35.07\%) have become the main pathogens involved in MDRO infections in the ICU, which is in line with the current epidemic trend. The proportion of MRDO infections accounted for by CRAB infections in this study was substantially different from that in Cui Congxian's study $^{20}$ (CRAB accounted for 20.44\%) in 2017; the MDROs in that study were mainly ESBL-producing Enterobacteriaceae (the proportion of ESBL-producing Enterobacteriaceae was relatively high due to the widespread use of the third-generation cephalosporins). In the present study, rates differed from the corresponding data of the National Bacterial Resistance Surveillance Network 
A

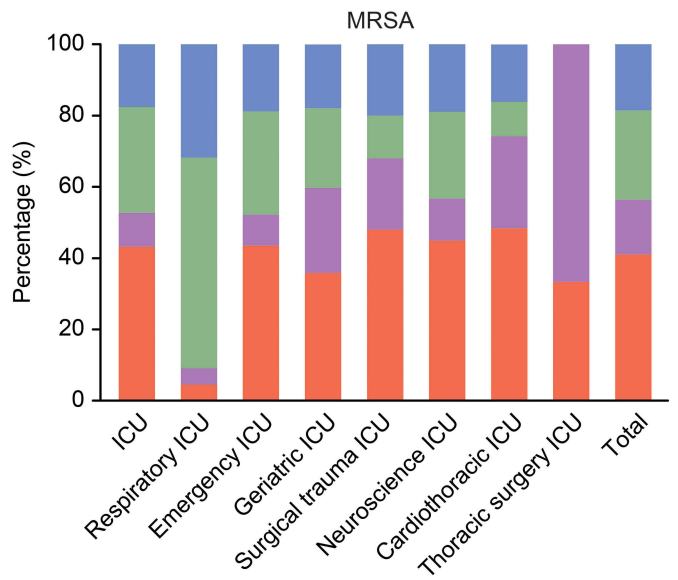

Department acquisition Out-of-hospital transfer In-hospital transfer Community acquisition

\section{C}

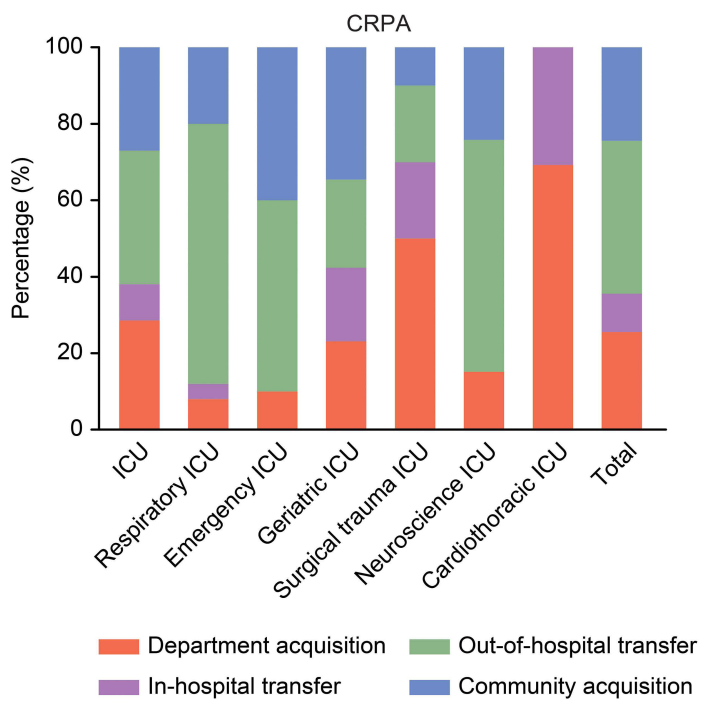

B

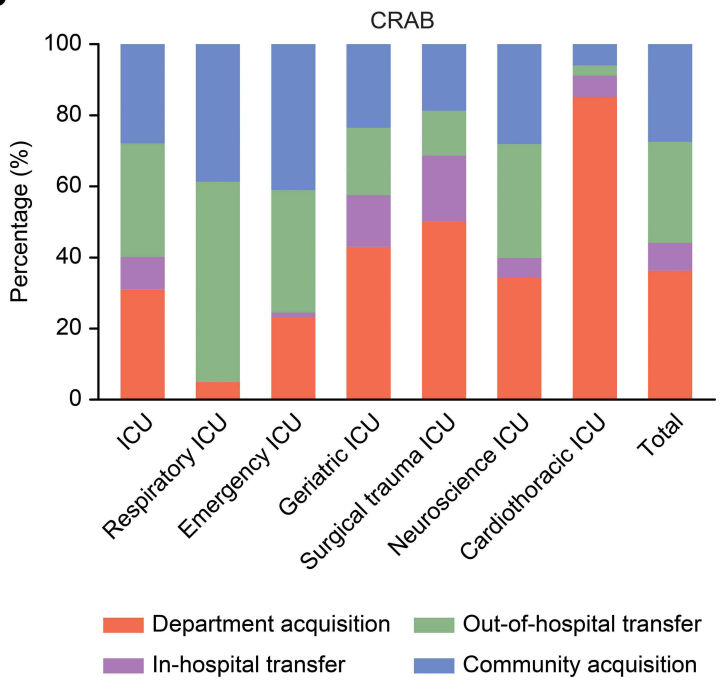

D

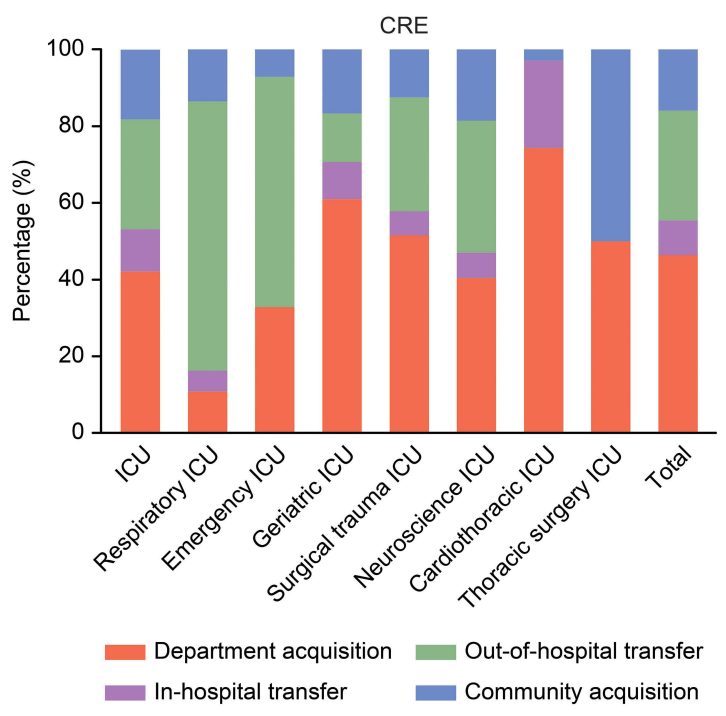

Figure 3 Source composition ratio of MDRO infection strains in 8 ICU wards. (A) Distribution of MRSA sources in 8 different ICUs; (B) distribution of CRAB sources in 8 different ICUs; (C) distribution of CRPA sources in 8 different ICUs; (D) distribution of CRE sources in 8 different IC.

(CARSS) ICU in $2017 ;^{21}$ the MDROs in the 8 ICU wards in our study were MRSA (61.24\%), CRAB (83.75\%), CRPA (43.01\%) and CRE (30.15\%), which were higher than those in CARSS in 2017 [MRSA (45.60\%), CRAB (81.90\%), CRPA (35.80\%) and CRE (20.60\%)]. The main reason is that in CARSS, the data provided are the national averages; however, the detection rate of MDROs in Jiangsu Province is the highest in the country. Our hospital is the best comprehensive hospital in Jiangsu Province, and the vulnerable population with MDRO infections, such as patients in the ICU, is consequently relatively larger. However, these data still reflect to some extent that the detection rate of gram-negative bacilli among MDROs, especially those resistant to carbapenem antibiotics, is relatively high in the ICU of our hospital. Thus, we need to continue to strengthen the strategies for the prevention and control of MDROs.

Among MDRO infection sites, lower respiratory tract infection $(81.10 \%)$ was most common, and the proportion of lower respiratory tract infections was higher than that reported in related studies in other countries $(64.00 \%){ }^{22}$ which is associated with the serious condition of ICU inpatients, greater use of ventilators, long duration of hospitalization, and long duration of bed rest, among 
Table 2 Comparison Between Sources of MDRO Infection in the Comprehensive ICU and the Specialized ICU (n, \%)

\begin{tabular}{|c|c|c|c|c|c|}
\hline MDRO & Source & Comprehensive ICU & Specialized ICU & $\chi^{2}$ & $\mathbf{P}$ \\
\hline MRSA $(n=427)$ & $\begin{array}{l}\text { Out-of-hospital transfer } \\
\text { Community acquisition } \\
\text { In-hospital transfer } \\
\text { Department acquisition }\end{array}$ & $\begin{array}{l}57(27.14) \\
38(18.10) \\
29(13.81) \\
86(40.95)\end{array}$ & $\begin{array}{l}50(23.04) \\
41(18.89) \\
37(17.05) \\
89(41.01)\end{array}$ & 1.479 & 0.687 \\
\hline CRAB $(n=1100)$ & $\begin{array}{l}\text { Out-of-hospital transfer } \\
\text { Community acquisition } \\
\text { In-hospital transfer } \\
\text { Department acquisition }\end{array}$ & $\begin{array}{l}204(28.77) \\
209(29.48) \\
64(9.03) \\
232(32.72)\end{array}$ & $\begin{array}{l}109(27.88) \\
93(23.79) \\
22(5.63) \\
167(42.71)\end{array}$ & 13.705 & 0.003 \\
\hline CRPA $(n=180)$ & $\begin{array}{l}\text { Out-of-hospital transfer } \\
\text { Community acquisition } \\
\text { In-hospital transfer } \\
\text { Department acquisition }\end{array}$ & $\begin{array}{l}33(33.33) \\
30(30.30) \\
11(11.11) \\
25(25.25)\end{array}$ & $\begin{array}{l}39(48.15) \\
\mid 4(17.28) \\
7(8.64) \\
2 \mid(25.93)\end{array}$ & 5.813 & 0.121 \\
\hline CRE $(n=922)$ & $\begin{array}{l}\text { Out-of-hospital transfer } \\
\text { Community acquisition } \\
\text { In-hospital transfer } \\
\text { Department acquisition }\end{array}$ & $\begin{array}{c}167(26.38) \\
104(16.43) \\
59(9.32) \\
303(47.87)\end{array}$ & $\begin{array}{c}97(33.56) \\
43(14.88) \\
24(8.30) \\
125(43.25)\end{array}$ & 5.011 & 0.171 \\
\hline $\begin{array}{l}\chi^{2} \\
P\end{array}$ & & $\begin{array}{l}60.014 \\
<0.001\end{array}$ & $\begin{array}{l}46.549 \\
<0.001\end{array}$ & & \\
\hline
\end{tabular}

other factors. Therefore, more attention should be given to strengthening the measures to prevent and control respiratory tract infections in ICU patients. At the same time, the high rates may be related to the fact that clinicians send more sputum samples for examination, and because there is a large amount of pathogenic bacteria in the respiratory tract, contamination can easily occur when the samples are collected; hence, the significance of sending sputum samples for examination remains to be clarified. ${ }^{23}$

Most studies have reported that the ICU is the source of MDROs, and it has thus become a key department for MDRO prevention and control. The rates of out-ofhospital and in-hospital MDRO infections in the ICU ward were $50.51 \%$ and $49.49 \%$, respectively, in this study. More than half of the MDRO infections were outof-hospital infections (out-of-hospital transfer or community acquired), with MRSA, CRAB, CRPA and CRE proportions of $43.56 \%, 55.91,64.44 \%$ and $44.58 \%$, respectively; however, MDRO infection rates of acquisition in the department were only $40.98 \%, 36.27 \%, 25.56 \%$ and $46.62 \%$, respectively. Indeed, more than $50 \%$ of MDRO infections in the ICU ward were imported. Even if all MDRO infections acquired in the ICU could be eliminated, the "overflow" of MDRO infections into the ICU ward would still not be resolved because the sources would not be controlled. At present, the majority of domestic ICUs do not identify (such as by active screening) and control the sources of imported MDRO infections, which is why domestic ICUs have become a focus of attention, though the MDRO infection problem has not yet improved significantly. The high rate of transfers from outside hospitals and community sources indicates that bacterial resistance in lower-level hospitals and communities is relatively high, which should be given sufficient attention by the health administration.

At the national level, the management of MDRO prevention and control is no longer merely the responsibility of a hospital but of the entire society. To address the issue of MDRO infection at a deeper level, relevant departments should implement plans from the top level to improve the rational use of antimicrobial drugs in lower-level hospitals and strengthen the management of antimicrobial drug use in industry. At the same time, at the hospital level, the ICU is still the key target of MDRO infection prevention and control. However, ICU management needs to be further refined, and targeted prevention and control should be conducted according to the characteristics of the sources of MDRO infection in each ICU. Only when hospitals and society are linked together can we better build a barrier to fight against bacterial resistance and protect human health.

Acinetobacter baumannii is a nonfermenting gramnegative bacillus that is pathogenic to humans. It is widely 
distributed in the hospital environment and has a long survival time; it also has natural resistance to a variety of antibacterial drugs. For example, resistance to carbapenems has become increasingly serious due to the widespread application of these drugs, attracting extensive attention from researchers at home and abroad. ${ }^{24}$ In addition, the comparison of the comprehensive and specialized ICUs in this study showed significant differences in the proportions of CRAB infections that were community acquired, acquired elsewhere in the hospital and acquired in the ICU. With regard to CRAB infections, the comprehensive ICU had a higher proportion of communityacquired and in-hospital infections and a lower proportion of ICU-acquired infections than the specialized ICU. Therefore, in terms of CRAB infection prevention and control in comprehensive ICUs, more attention should be paid to sources in the community and other wards of the hospital. In specialized ICUs, CRAB infections acquired in the department should be a topic of focus.

This research involves a retrospective design, and there are still some limitations. First, some cases were determined based on the detection time of MDRO, which is somewhat different from the actual time. Second, when determining the specific source of MDRO in the ICU ward, it can only be extracted based on archived case data, and incomplete records or missing data may be a constraint. In the future, we will continue to carry out multicenter studies and prospective research to further expand on our findings.

\section{Conclusion}

Different sources of MDRO vary in different ICUS.The main source of MDROs in the ICU is not from department acquisition itself entirely, and a large proportion of MDRO sources in ICU units are imported (including: out-hospital transfer, community acquisition and in-hospital transfer).It is particularly important to strengthen the identification of MDRO sources and implement more effective and accurate infection prevention and control measures.

\section{Ethics Statement}

The authors are accountable for all aspects of the work with regard to ensuring that questions related to the accuracy or integrity of any part of the work are appropriately investigated and resolved. This study was approved by Institutional Review Board- First Affiliated Hospital of Nanjing Medical University (No. 2019-SR-075), and each hospital received permission to participate in this study and sign a cooperation agreement. Written informed consent was obtained from all patients. This study was conducted in accordance with the Declaration of Helsinki.

\section{Consent for Publication}

We had obtained from the patient for written informed consent for publication.

\section{Acknowledgments}

We thank Xing $\mathrm{Wu}$ and Ge Song for their contributions to data collection in this research.

\section{Author Contributions}

All authors made substantial contributions to conception and design, acquisition of data, or analysis and interpretation of data; took part in drafting the article or revising it critically for important intellectual content; agreed to submit to the current journal; gave final approval of the version to be published; and agree to be accountable for all aspects of the work.

\section{Funding}

This project was supported by the National Key R \& D Program Fund (2020YFC0848100), the Research Foundation for Infection Prevention and Control of Chinese Geriatrics Association (GRYJ-LRK2018016), Top Talent Support Program for young and middle-aged people of Wuxi Health Committee (No. HB2020040), Mega-project of Wuxi Commission of Health (No. Z202007), and the Jiangsu Provincial Hospital Management Innovation Research Fund (JSYGY-3-2019484).

\section{Disclosure}

The authors have no conflicts of interest to declare.

\section{References}

1. Vanholder R, Van Biesen W, Lameire N. What is the renal replacement method of first choice for intensive care patients. $J$ Am Soc Nephrol. 2001;12(Suppl 17):S40-S43. doi:10.1681/ASN.V12suppl_1s40

2. Russotto V, Cortegiani A, Graziano G, et al. Bloodstream infections in intensive care unit patients: distribution and antibiotic resistance of bacteria. Infect Drug Resist. 2015;8:287-296. doi:10.2147/IDR. S48810

3. Chen Y, Wang C, Chen L. Multidisciplinary cooperation model for prevention and control of ICU MDRO infection [Chinese]. Chin $J$ Hosp Epidemiol. 2018;28(18):2839-2842.

4. Cassone M, Mody L. Colonization with multi-drug resistant organisms in nursing homes: scope, importance, and management. Curr Geriatr Rep. 2015;4:87-95. doi:10.1007/s13670-015-0120-2 
5. Davies J, Davies D. Origins and evolution of antibiotic resistance. Microbiol Mol Biol Rev. 2010;74:417-433. doi:10.1128/MMBR.0001610

6. Hidron AI, Edwards JR, Patel J, et al. Antimicrobial-resistant pathogens associated with healthcare-associated infections: annual summary of data reported to the national healthcare safety network at the centers for disease control and prevention, 2006-2007. Infect Control Hosp Epidemiol. 2008;29:996-1011. doi:10.1086/591861

7. Vincent JL, Sakr Y, Sprung CL, et al. Sepsis in European intensive care units: results of the SOAP study. Crit Care Med. 2006;34:344-353. doi:10.1097/01.CCM.0000194725.48928.3A

8. Maragakis LL. Recognition and prevention of multidrug-resistant Gram-negative bacteria in the intensive care unit. Crit Care Med. 2010;38:S345-S351. doi:10.1097/CCM.0b013e3181e6cbc5

9. Peleg AY, Hooper DC. Hospital-acquired infections due to gram-negative bacteria. $N$ Engl J Med. 2010;362:1804-1813. doi:10.1056/NEJMra0904124

10. Arias CA, Murray BE. Antibiotic-resistant bugs in the 21 st century a clinical super-challenge. $N$ Engl J Med. 2009;360:439-443. doi:10.1056/NEJMp0804651

11. Zhou X, Du G, Li Y, Chen X, Qu H. Detection and drug resistance of MDRO in ICU and non-ICU wards [Chinese]. Chin J Infect Control. 2018; 17:219-223+229.

12. Vincent JL, Marshall JC, Namendys-silva SA, et al. Assessment of the worldwide burden of critical illness: the Intensive Care Over Nations (ICON) audit. Lancet Respir Med. 2014;2:380-386. doi:10.1016/S2213-2600(14)70061-X

13. Di Gaudio F, Indelicato S, Indelicato S, Tricoli MR, Stampone G, Bongiorno D. Improvement of a rapid direct blood culture microbial identification protocol using MALDI-TOF MS and performance comparison with SepsiTyper kit. $J$ Microbiol Methods. 2018;155:1-7. doi:10.1016/j.mimet.2018.10.015

14. (CLSI) Clinical and Laboratory Standards Institute. Performance Standards for Antimicrobial Susceptibility Testing, Twenty Seventh Informational Supplement. M100S, 27th ed. (CLSI) Clinical and Laboratory Standards Institute; 2017.

15. Ministry of Health of the People's Republic of China. Diagnostic criteria of nosocomial infection (trial). Chin Med J. 2001;(05):61-67.

16. Magiorakos AP, Srinivasan A, Carey RB, et al. Multidrug-resistant, extensively drug-resistant and pandrug-resistant bacteria: an international expert proposal for interim standard definitions for acquired resistance. Clin Microbiol Infect. 2012;18:268-281. doi:10.1111/ j.1469-0691.2011.03570.x
17. Russotto V, Cortegiani A, Fasciana T, et al. What healthcare workers should know about environmental bacterial contamination in the intensive care unit. BioMed Res Int. 2017;2017:6905450. doi:10.1155/2017/6905450

18. Li F, Yang Y, Liu C, et al. Analysis of infection status and risk factors of multidrug resistant bacteria in ICU [Chinese]. Chin J Hosp Epidemiol. 2016;783-785. Available from: https://kns.cnki.net/kcms/detail/detail. asp $\mathrm{x}$ ?dbcode $=\mathrm{CJFD} \& \mathrm{dbname}=\mathrm{CJFDLAST} 2016 \&$ filename $=$ ZHYY201604025\&uniplatform=NZKPT\&v=LkDFIU42 LdJnOxhd qW1epcLsHsVvbRizUTellR9kP1FFlGM_X_QA4kfTwClzEE. Accessed November 22, 2021.

19. Hu F, Guo Y, Zhu D, et al. Surveillance of bacterial drug resistance in China by CHINET in 2017 [Chinese]. Chin J Infect Chemother. 2018. Available from: https://kns.cnki.net/kcms/detail/detail.aspx?dbcode= C J F D \& d b n a m e = C J F D L A S T 2018 \& fi 1 e n a m e = KGHL201803001\&uniplatform=NZKPT\&v=59T2O-YB7wothcTidTPKS3wWecSnGYR8PtPvDTDT5arrcrIDCcZWH3aeTE_ Myhi. Accessed November 22, 2021.

20. Cui C, Li Q, Tong H, et al. Clinical analysis and drug resistance surveillance of multidrug resistant bacteria infection in intensive care inpatients [Chinese]. Chin J Hosp Epidemiol. 2017;27:2430-2433.

21. National bacterial Drug Resistance Surveillance Network (CARSS). National bacterial drug resistance surveillance report; 2017. Available from: http://www.carss.cn/Report/Details?aId=552. Accessed November 19, 2021

22. Zilahi G, Artigas A, Martin-Loeches I. What's new in multidrugresistant pathogens in the ICU? Ann Intensive Care. 2016;6:96. doi:10.1186/s13613-016-0199-4

23. Zhang Y, Gong R, Liu Z, et al. Analysis of 677 cases of nosocomial infection caused by multidrug resistant bacteria. Chin $J$ Hosp Epidemiol. 2017. Available from: https://kns.cnki.net/ $\mathrm{kcms} / \mathrm{detai} 1 / \mathrm{detail}$. as px ? dbcode $=$ C JFD \& dbname CJFDLAST2017\&filename=ZHYY201718010\&uniplatform= N Z K P T \& v $=1$ E M y D 8 y 0 s 591 x 7 x C b m C S p h z if 0 cBjWzWcEOMTAT2nE2k604eI8BwKngHwzFQyF5. Accessed November 22, 2021.

24. Chittawatanarat K, Jaipakdee W, Chotirosniramit $\mathrm{N}$, et al. Microbiology, resistance patterns, and risk factors of mortality in ventilator-associated bacterial pneumonia in a Northern Thai tertiary-care university based general surgical intensive care unit. Infect Drug Resist. 2014;7:203-210. doi:10.2147/IDR.S67267
Infection and Drug Resistance

\section{Publish your work in this journal}

Infection and Drug Resistance is an international, peer-reviewed openaccess journal that focuses on the optimal treatment of infection (bacterial, fungal and viral) and the development and institution of preventive strategies to minimize the development and spread of resistance. The journal is specifically concerned with the epidemiology of antibiotic resistance and the mechanisms of resistance development and diffusion in both hospitals and the community. The manuscript management system is completely online and includes a very quick and fair peerreview system, which is all easy to use. Visit http://www.dovepress.com/ testimonials.php to read real quotes from published authors. 